Ann. Abeille, I967, 10 (I), 39-44.

\title{
ÉTUDE (A L'AIDE D'UN RADIO-ISOTOPE) DE LA TROPHALLAXIE CHEZ L'ABEILLE PENDANT L'ÉTÉ
}

\author{
P. DOUAULT \\ Station de Recherches sur l'Abeille et les Insectes sociaux, 91 -Bures-sur-Yvette
}

\section{SOMMAIRE}

L'auteur étudie les échanges de nourritures entre abeilles ouvrières à l'intérieur d'une ruche expérimentale au cours des mois d'avril à août.

Ces recherches sont faites avec l'aide d'un radioisotope : l'or I98 ((Au 198).

Les résultats mettent en évidence l'influence du couvain sur les échanges et les modifications qui interviennent lorsqu'il y a absence de couvain, mais malgré tout présence de la reine.

\section{IN'TRODUC'TION}

Différents auteurs ont déjà étudié les échanges de nourriture chez 1'Abeille à l'intérieur d'une ruche.

Nixon et RibBands (I952) ont distribué un sirop de sucre radioactif à une colonie et ont pu constater qu'au bout de 24 heures plus de la moitié de la ruche était contaminée.

OERTEL, et al. (I953) ont observé les échanges entre mâles et ouvrières au moyen d'un radioisotope.

COURTOIS et LECOMTE (I958) ont constaté qu'après ingestion d'or I 98 , au bout de 36 heures, Ioo p. Ioo des abeilles appartenant à une même ruche devenaient radioactives.

COURTOIS, I.ECOMTE et SALLERON (I96I) ont étudié les échanges de nourriture entre abeilles ouvrières, mais seulement pendant la période hivernale, au cours des mois de décembre et janvier. 
Pour leurs recherches, ces derniers auteurs ont utilisé une ruche expérimentale dite " ruche de Chauvin ". Cette ruche comprenait un cadre unique dont les dimensions étaient de $\Upsilon_{40} \times$ Ioo $\mathrm{cm}$, logé dans une armoire chauffée aux parois vitrées afin que les observations soient possibles de l'extérieur.

Les résultats de leurs travaux ont mis en évidence le comportement d'échanges des ouvrières au sein de "la grappe " hivernale à une période où il n'y a pas de couvain.

Ils ont utilisé pour le marquage 1 'Au I98; et la nourriture marquée à base de miel, était administrée à une dizaine d'abeilles qui rentraient aussitôt après ingestion de cette nourriture en communication avec le reste de la population.

Nous inspirant de ce travail, nous avons voulu étudier les échanges au cours de la belle saison en commençant nos expériences au mois d'avril et les poursuivant jusqu'à la fin août.

Nous avons recherché : Io l'influence que pouvait avoir le nid à couvain sur les échanges entre abeilles; $2^{\circ}$ l'influence de la reine sur les échanges.

\section{MATÉRIEL, ET MÉTHODES}

\section{La ruche.}

La ruche expérimentale utilisée était du type "ruche de Chauvin " mais avec des dimensions plus réduites. Le cadre mesurait $95 \mathrm{~cm}$ de largeur sur $85 \mathrm{~cm}$ de hauteur. La feuille de cire gaufrée était appliquée directement sur un panneau opaque de façon à ce que les abeilles ne puissent construire qu'une seule face. Le panneau était lui-même compris entre deux plaques de Plexiglas entouré d'un cadre en bois pour maintenir le tout. L'ensemble se trouvait logé dans une armoire vitrée, formant étuve, chauffée par une résistance, commandée par un thermostat d'ambiance de précision, maintenant une température constante de $30^{\circ} \mathrm{C}$.

La ruche était en communication avec l'extérieur permettant la libre sortie des abeilles.

\section{La population.}

Les abeilles introduites dans la ruche étaient de race jaune, d'origine italienne. La colonie comptait environ 3000 individus répartis sur le cadre.

\section{Technique de marquage.}

L'isotope utilisé était l'or I98 (Au 198) préparé par le C. E. A. (Saclay) et présenté sous la forme d'or colloïdal. Ce radio-élément a été choisi à cause de sa facilité de détection, son émission $\gamma$ étant de $0,4 \mathrm{II} \mathrm{MeV}$, et également de sa période courte d'une durée de 2,7 jours.

\section{Marquage des abeilles.}

A chaque expérience, une seule abeille était marquée. L'abeille destinée à faire les premiers échanges était prélevée au trou de vol ; nous avions ainsi toutes les chances de choisir une butineuse et de nous mettre le plus près possible des conditions naturelles.

L'abeille était nourrie seule dans une cagette, avec une nourriture miellée contenant le radioisotope, contrôlée avec l'appareil de détection et aussitôt après déposée à l'orifice d'envol afin de lui permettre de rentrer dans la ruche.

\section{Méthode de détection.}

L'appareil que nous avons utilisé était un détecteur portatif à scintillation S. R. A. T., S. P. P. 2 à lecture directe. 
La surface vitrée de la ruche était divisée en ${ }_{15} 6$ carrés de $6 \mathrm{~cm}$ de côté. La surface de chaque carré était couverte par l'élément sensible du scintillateur et la détection se trouvait faite d'une manière particulièrement efficace.

\section{RÉSULTATS}

Nous avons procédé à des essais pendant deux années consécutives et comparé les réstultats.

Le premier essai a été effectué au mois de juin I965 alors que le nid à couvain occupait tout le centre de la ruche (fig. I $a$ ). Aussitôt, après avoir été nourrie au miel marqué, l'abeille donneuse s'est dirigée, à partir du trou de vol, en direction du nid à couvain et dans sa partie supérieure ; la ligne pointillée montre la trajectoire des échanges avant leur concentration sur une certaine aire. Comme l'indique la figure, la distribution entre les abeilles s'est effectuée sur toute la surface du couvain operculé et s'est accumulée à la base de celui-ci en s'étendant sur une aire contenant des cellules occupées par des larves en cours d'élevage.

Moins de 30 minutes après l'introduction de l'abeille donneuse dans la ruche, les échanges s'effectuaient sur le nid à couvain et en l'espace d'une heure s'étendaient à toute sa surface. Après s'être concentrés sur le couvain ouvert, les échanges devaient s'y effectuer jusqu'à la fin de l'expérience, celle-ci ayant débutée le matin à 9 h 30 , se poursuivait jusqu'au soir. Le lendemain, au cours d'un contrôle, nous remarquions que la radioactivité était toujours concentrée sur la même surface que la veille.

Un essai effectué le 3 aô̂t 1965 , nous a apporté des résultats analogues. I e couvain à cette époque se trouvait dans une autre partie de la ruche et le " nid » était disposé parallèlement à un des montants du cadre (fig I $b$ ) et divisé en deux plaques.

L'abeille donneuse, après son introduction dans la colonie, s'est dirigée immédiatement vers la partie supérieure du nid pour commencer ses échanges.

Les communications entre abeilles se sont fortement intensifiées dans une zone séparant les deux plaques de couvain et se sont étendues à une autre partie du nid. Les échanges ont été constatés pendant toute la durée de l'expérience.

Le Io aô̂t I 965 , nous avons procédé à un autre essai, alors que le couvain. occupait sensiblement la même place que pendant la précédente expérience (fig. I $c$ ),

Une fois introduite dans la ruche, l'abeille porteuse de la radioactivité a commencé ses premiers échanges sur la partie supérieure du nid à couvain où ils se sont poursuivis et 30 minutes environ après le début de l'expérience, la surface totale du couvain était radioactive. Dans tous les cas énumérés, la radioactivité détectée sur les surfaces éloignées du couvain était infime ou quasi inexistante.

L'année suivante, afin de ne pas utiliser la même colonie d'abeilles, nous avons procédé à un nettoyage de la ruche, changé la cire et apporté une population nouvelle. Cette population comptait un nombre d'abeilles à peu près équivalent au précédent.

Au mois d'avril I g66, la nouvelle colonie avait entièrement construit la cire et le nid à couvain étant suffisant, nous avons repris nos expériences (fig. I $d$ ).

Un essai le 24 avril nous a permis de constater les mêmes phénomènes qu'en Ig65. L'abeille donneuse, dès son entrée dans la ruche, a fait une rapide exploration 

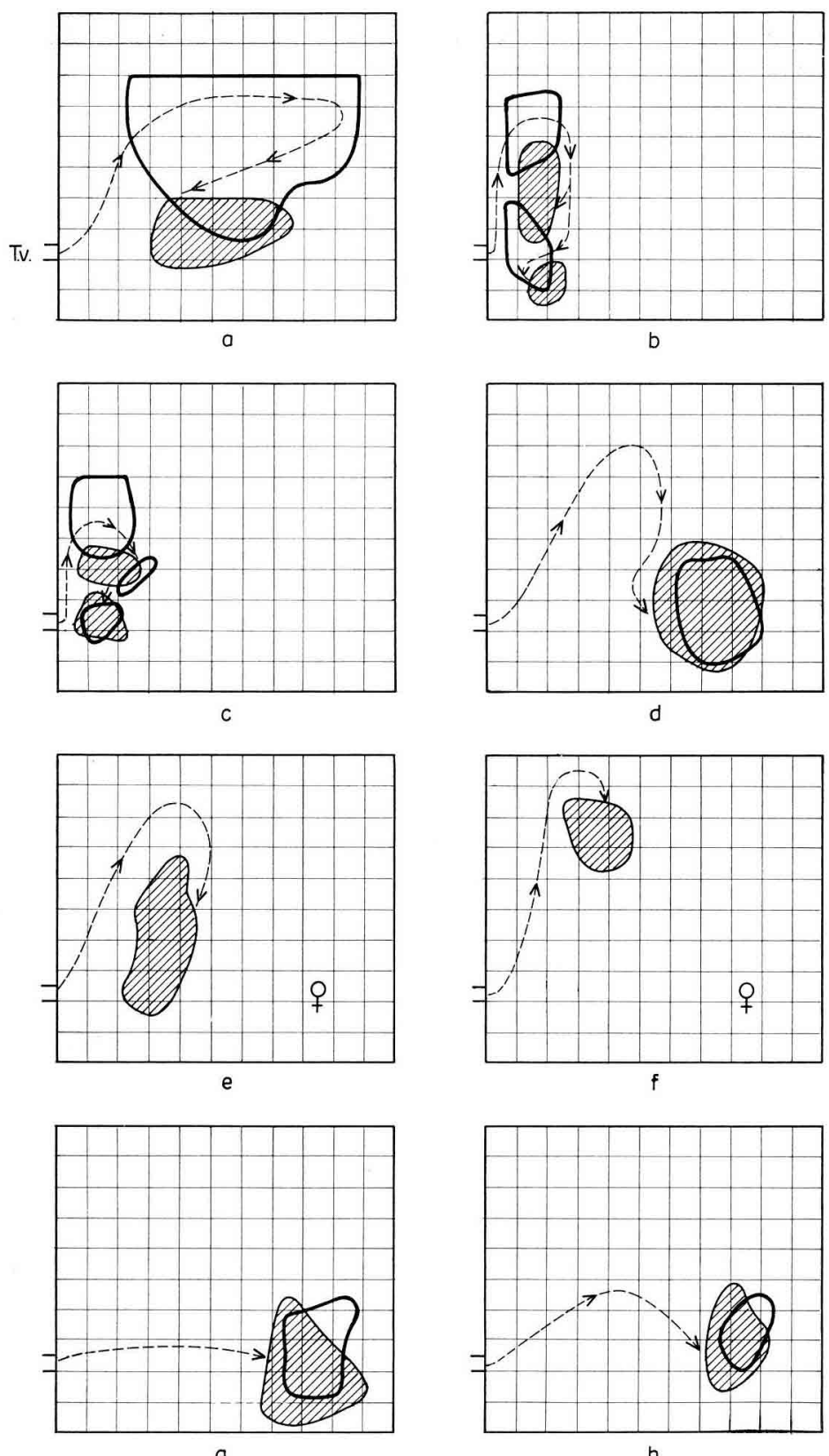

g

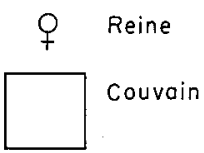

\section{Concentration de} la radioactivité

FIG. I. - Répartition de la radioactivité par rapport au nid d couvain dans les expériences successives 
de la partie supérieure du cadre en procédant à de rares échanges et s'est dirigée rapidement vers le nid à couvain, oì les rapports entre abeilles se sont intensifiés de telle façon que 3 heures après le début de l'expérience, le nid à couvain se trouvait totalement englobé dans une zone radioactive.

Tenant compte de tous ces résultats, nous avons tout de suite pensé à l'influence de la reine sur les échanges. La reine se tient toujours à proximité du couvain et même se trouve généralement au milieu du nid lors de la formation de la grappe hivernale.

Profitant d'une période où la reine avait interrompu sa ponte et où tout le couvain était né, nous avons procédé à deux essais.

Le premier, réalisé le 5 mai, nous a permis de constater que les échanges ne s'effectuaient pas vers la reine ou dans son voisinage. L'abeille donneuse après une courte exploration accompagnée de quelques échanges a intensifié ses rapports avec ses congénères dans une zone très éloignée de la reine. Celle-ci se tenait sur son ancien lieu de ponte entourée de sa " cour " habituelle (fig. I $e$ ). Tout au long de l'expérience nous n'avons jamais remarqué d'échanges en direction de la reine.

Un deuxième essai réalisé le $\mathrm{x} 2$ mai, la ruche se trouvant dans des conditions semblables, nous a apporté les mêmes résultats (fig. I f). Encore une fois, les échanges se sont effectués dans une autre partie de la ruche et dans un lieu éloigné de la reine sans jamais se rapprocher d'elle.

Un peu plus tard, nous avons remplacé à nouveau la cire et la colonie et attendu que la reine commence sa ponte. Une fois le couvain operculé nous avons fait deux essais au mois d'août Ig66.

Ces deux essais (fig. I $g$ et $h$ ) nous ont donné les mêmes résultats que ceux enregistrés l'année précédente.

Dans les deux cas l'abeille donneuse a procédé à quelques échanges en direction du couvain et très rapidement les communications entre abeilles s'opéraient sur le nid et dans son étroit voisinage.

\section{CONCLUSION}

Cette série d'expériences, conduite pendant deux années consécutives nous a apporté, pensons-nous, des renseignements intéressants sur le déterminisme de certains phénomènes sociaux chez l'Abeille.

Il nous est permis de penser que le couvain joue un rôle important dans le comportement des abeilles et que les échanges trophallactiques s'en trouvent influencés d'une façon certaine.

La présence de la reine ne semble pas agir directement sur les communications entre les insectes, tout au moins pendant cette période de l'année où la colonie se trouve beaucoup plus dispersée que pendant la saison hivernale. 


\section{SUMMARY}

\section{RADIOISOTOPE STUDY OF TROPHALLAXY BETWEEN BEES IN SUMMER}

Food exchanges between worker bees were studied in an experimental hive from April to August. In all the cases enumerated the radioactive zone coincided with the broodcomb nest, the radioactivity detected on the remote surfaces of the brood being minute or almost non-existent. It was shown by experiments that the exchanges are not made towards the queen or near her.

\section{RÉFÉRENCES BIBLIOGRAPHIQUES}

Courtors G., Lecomte J., i958. Sur un procédé de marquarge des abeilles butineuses au noven d'un radioisotope. C. R. Acud. Sci., 247, 147 -1 49 .

Courtors G., Leconte J., Salleron Fr., ighor. Étude des échanges de nourriture à l'intérieur de la ruche entre les abeilles ouvrières (Apis mellifica I.) C. R. Acad. Sci., 252, 1057-1059.

Nixon H. L., Ribbands C. R., $195^{2}$. Food transmission within the honeybee community. Proc. $r$. Soc. London, 140, 43-50.

Oertel E., Emersox R. B., Wheeler II. E., 1953. Transfer of radioactivity from worker to drone honey bees after ingestion of radioactive sucrose. Amn. entomol. Soc. Amer., $\mathbf{4 6}(4), 516-598$. 